\title{
The Measurement of the Sensory Recovery Period in Zygoma and Blow-Out Fractures with Neurometer Current Perception Threshold
}

\author{
Daemyung $\mathrm{Oh}^{1}$, Taebin Yun ${ }^{1}$, Junhyung $\mathrm{Kim}^{1}$, Jaehoon $\mathrm{Choi}^{1}$, Woonhyeok Jeong ${ }^{1}$, Hojun $\mathrm{Chu}^{1}$, \\ Soyoung Lee ${ }^{2}$ \\ Departments of ${ }^{1}$ Plastic and Reconstructive Surgery and ${ }^{2}$ Rehabilitation Medicine, Keimyung University Dongsan Medical Center, \\ Keimyung University School of Medicine, Daegu, Korea
}

Background Facial hypoesthesia is one of the most troublesome complaints in the management of facial bone fractures. However, there is a lack of literature on facial sensory recovery after facial trauma. The purpose of this study was to evaluate the facial sensory recovery period for facial bone fractures using Neurometer.

Methods Sixty-three patients who underwent open reduction of zygomatic and blowout fractures between December 2013 and July 2015 were included in the study. The facial sensory status of the patients was repeatedly examined preoperatively and postoperatively by Neurometer current perception threshold (CPT) until the results were normalized.

Results Among the 63 subjects, 30 patients had normal Neurometer results preoperatively and postoperatively. According to fracture types, 17 patients with blowout fracture had a median recovery period of 0.25 months. Twelve patients with zygomatic fracture had a median recovery period of 1.00 month. Four patients with both fracture types had a median recovery period of 0.625 months. The median recovery period of all 33 patients was 0.25 months. There was no statistically significant difference in the sensory recovery period between types and subgroups of zygomatic and blowout fractures. In addition, there was no statistically significant difference in the sensory recovery period according to Neurometer results and the patients' own subjective reports.

Conclusions Neurometer CPT is effective for evaluating and comparing preoperative and postoperative facial sensory status and evaluating the sensory recovery period in facial bone fracture patients.

Keywords Zygomatic fractures / Orbital fractures / Hypesthesia
Correspondence: Junhyung Kim Department of Plastic and Reconstructive Surgery, Keimyung University Dongsan Medical Center, Keimyung University School of Medicine, 56 Dalseong-ro, Jung-gu, Daegu 41931, Korea

Tel: +82-53-250-7635

Fax: +82-53-255-0632

E-mail: med69@dsmc.or.kr

This article was presented at the 73rd Congress of the Korean Society of Plastic and Reconstructive Surgeons on November 13-15, 2015 on Seoul, Korea.

No potential conflict of interest relevant to this article was reported.

Received: 23 May 2016 • Revised: 9 Sep 2016 • Accepted: 13 Sep 2016

pISSN: 2234-6163 • elSSN: 2234-6171 • http://dx.doi.org/10.5999/aps.2016.43.5.411 • Arch Plast Surg 2016;43:411-417

\section{INTRODUCTION}

Facial bone fracture patients suffer from a variety of symptoms. Among these symptoms, pain, tenderness, and limitation in mouth opening can improve soon after open reduction. How- ever, symptoms that are triggered by trigeminal nerve injury, such as facial hypoesthesia, have a longer recovery period. Such neurologic symptoms are a challenge to surgeons for the following reasons. First, facial hypoesthesia is a subjective symptom, and hence it is difficult to quantify. In addition, there are usually 
no available data on the preoperative status of patients with facial bone fracture who suffer from facial hypoesthesia [1].

Current perception threshold (CPT) is the minimum amount of painless, neuroselective electrical stimulus that consistently elicits a neural response. The Neurometer generates a constant stimulus current by monitoring and compensating for variations in tissue impedance. The stimulus evokes responses that quantitatively measure the functional integrity of each of the three major sub-populations of sensory nerve fibers. Specifically, the A $\beta$, $\mathrm{A} \delta$, and $\mathrm{C}$ fiber groups are selectively stimulated by sinusoid waveform currents of $2,000 \mathrm{~Hz}, 250 \mathrm{~Hz}$, and $5 \mathrm{~Hz}$, respectively. Using small surface electrodes, this test generates discrete double-blinded CPT measurements representing minimum detectable current intensities for each fiber type. The Neurometer is applied in various fields: in clinical monitoring, to document sensory impairments at any cutaneous or mucosal site, determine the clinical severity of an impairment or disability, provide reliable readings for serial evaluations, and assess neurotoxicity or neuroprotective sensation; in outcome measurements, for the assessment of pharmaceutical efficacy/neurotoxicity, nerve blocks, response to therapeutic interventions, and nerve regeneration and recovery of function postsurgery/injury; in occupational testing, to assess neck and lower back injury, carpal tunnel syndrome, vibration neuropathy, neurotoxic exposure, and in epidemiological studies; and in laboratory research, to provide non-invasive neuroselective animal response testing [2].

Therefore, in this study, we evaluated the facial sensory recovery period among facial bone fracture patients who had facial hypoesthesia by using the Neurometer CPT (Neurotron, Baltimore, MD, USA).

\section{METHODS}

\section{Patients}

From December 2013 to July 2015, 63 patients who received

\section{Table 1. The demographic data of the study population}

\begin{tabular}{|lc|}
\hline Characteristic & No. of patients (\%) \\
\hline Sex & \\
Male & $22(66.7)$ \\
Female & $11(33.3)$ \\
Type of fracture & \\
Blowout & $17(51.5)$ \\
Zygoma & $12(36.3)$ \\
Both & $4(12.2)$ \\
Details of fracture & \\
Assault & $16(48.5)$ \\
Traffic accident & $8(24.2)$ \\
Fall & $8(24.2)$ \\
Sport & $1(3.1)$ \\
\hline
\end{tabular}

open reduction underwent Neurometer CPT examinations preoperatively and postoperatively. Thirty of the 63 patients had normal Neurometer CPT results preoperatively and postoperatively. Thirty-three of the 63 patients had sensory-impaired Neurometer CPT results. Patients in an unconscious state due to multiple trauma, in an uncooperative state, or with unstable vital signs were excluded from the study. Details of the demographics are presented in Table 1. During the follow-up period, patients did not take any medication, such as steroids, to relieve facial hypoesthesia.

\section{Methods}

Among facial-bone fracture patients, the status of the maxillary branch of the trigeminal nerve was evaluated by Neurometer CPT (Fig. 1). The evaluation was performed preoperatively and postoperatively at 1 week, 1 month, 3 months, and 6 months and, subsequently, at 3-month intervals, until the results were normalized.

The examination was performed in the supine position. The investigator applied conduction to the test-affected side first. The investigator applied 2 electrodes onto the patient's cheek along the maxillary branch of the trigeminal nerve. The electrodes were fixed with tape (Fig. 2). The investigator regulated stimulation from $0.001 \mathrm{~mA}$ to $10 \mathrm{~mA}$, and the intensity at which the patient could feel the stimulation was considered to be the threshold. Thresholds were checked at frequencies of $2 \mathrm{KHz}$, $250 \mathrm{~Hz}$, and $5 \mathrm{~Hz}$. After the thresholds were checked at each frequency, the same examination was performed independently for the opposite cheek. After the input of threshold values into Neuval software (Neurotron), the patient's trigeminal nerve status was scored from 0 to 10 . As 0 indicated a normal trigeminal nerve status and higher scores indicated severe symptoms, the

Fig. 1. The Neurometer CPT device

By stimulating the patient's affected site and checking the threshold, an investigator can diagnose the patient's peripheral nerve status. In this study, the trigeminal nerve was targeted. CPT, current perception threshold.

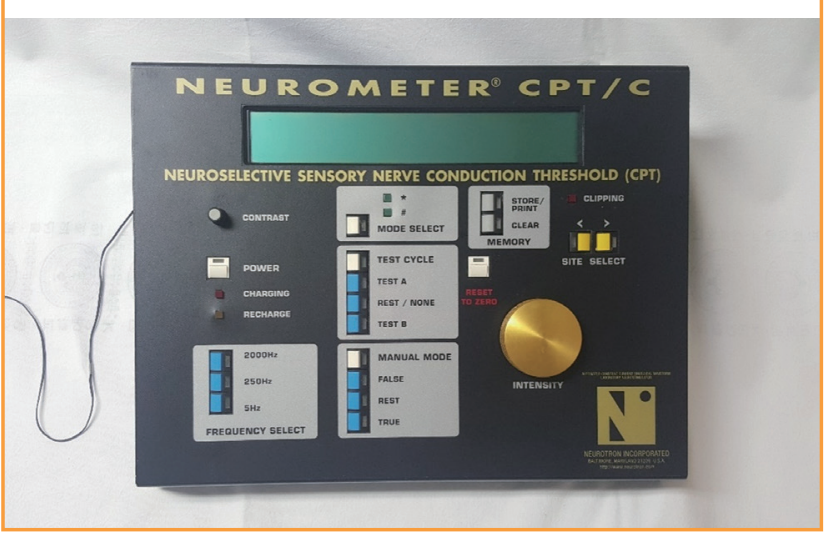


authors stopped performing follow-up when the patient's scores on both sides were 0 . The examination was carried out by a single rehabilitation physician.

The trigeminal nerve recovery period according to patients' own subjective feelings was determined based on a survey by direct conversation at the clinic or by phone call. If patients did not feel any hypoesthesia, the time was defined as the recovery period.

\section{Statistical analysis}

All data were analyzed with SPSS ver. 23.0 (IBM, Armonk, New York, USA). The recovery period of the test was evaluated. Differences in the recovery period of results according to the type

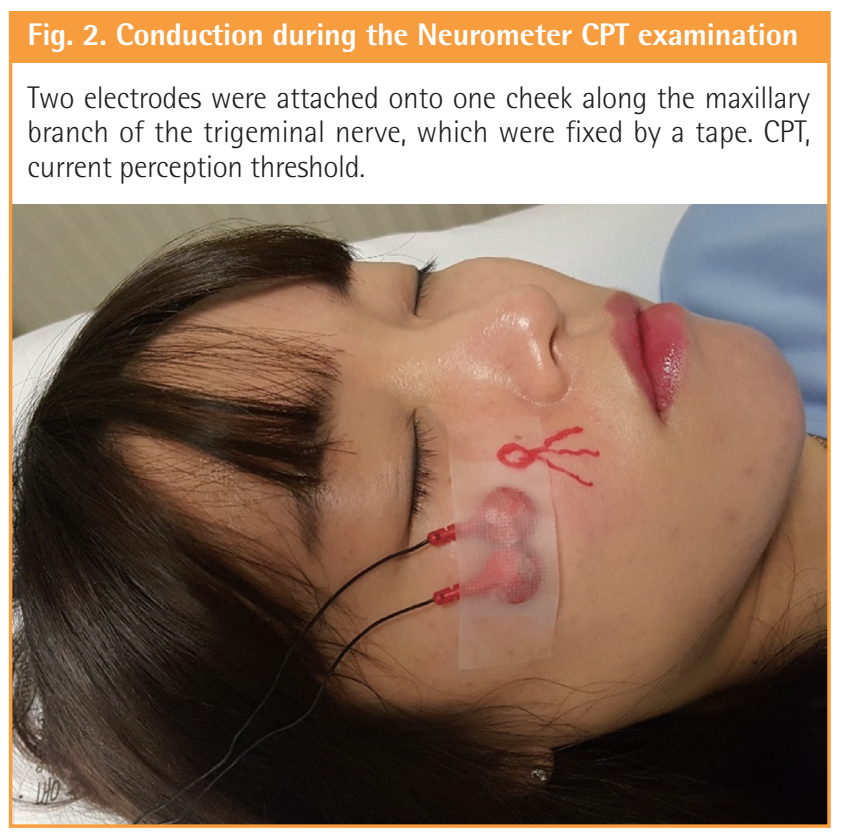

of fracture (fracture of the zygoma, blowout fracture, fracture of both types) were evaluated. Zygomatic fracture patients were classified according to Knight and North classification, and differences in the recovery period between classified groups were evaluated. Blowout fracture patients were classified according to the site of fracture (inferior wall, medial wall, and medioinferior wall), and differences in the recovery period among classified groups were evaluated. The Kruskal-Wallis test was used for comparison. The differences in the recovery period as assessed by Neurometer CPT examination and the recovery period according to patients' own subjective feelings were evaluated. The recovery period was obtained based on patients' feelings via a survey. A Wilcoxon signed-rank test was used for comparisons.

\section{RESULTS}

According to fracture types, 17 patients with blowout fracture had a median recovery period of 0.25 months. Twelve patients with zygomatic fracture had a median recovery period of 1.00 month. Four patients with both fracture types had a median re-

Table 2. The trigeminal nerve recovery period according to the type of fracture by Neurometer CPT test

\begin{tabular}{|lccc|}
\hline $\begin{array}{l}\text { Type of } \\
\text { fracture }\end{array}$ & $\begin{array}{c}\text { No. of } \\
\text { patients }\end{array}$ & $\begin{array}{c}\text { Trigeminal nerve } \\
\text { recovery period (mo) }\end{array}$ & P-value ${ }^{\text {a) }}$ \\
\hline Blowout & 17 & $0.25(0.25,6.00)$ & 0.468 \\
Zygoma & 12 & $1.00(0.25,14.00)$ & \\
Both & 4 & $0.625(0.25,3.00)$ & \\
Total & 33 & $0.25(0.25,14.00)$ & \\
\hline \multicolumn{4}{l}{$\begin{array}{l}\text { Values are presented as median (minimum, maximum). } \\
\text { CPT, current perception threshold. } \\
\text { a)Kruskal-Wallis test. }\end{array}$} \\
\hline
\end{tabular}

\section{Fig. 3. Knight and North classification of zygomatic fractures}

(A) Group III: unrotated body fractures; downward and inward displacement, but no rotation. (B) Group IV: medially rotated body fractures; downward, inward, and backward displacement with medial rotation. (C) Group V: laterally rotated body fractures; downward, backward, and medial displacement with lateral rotation of the zygoma.
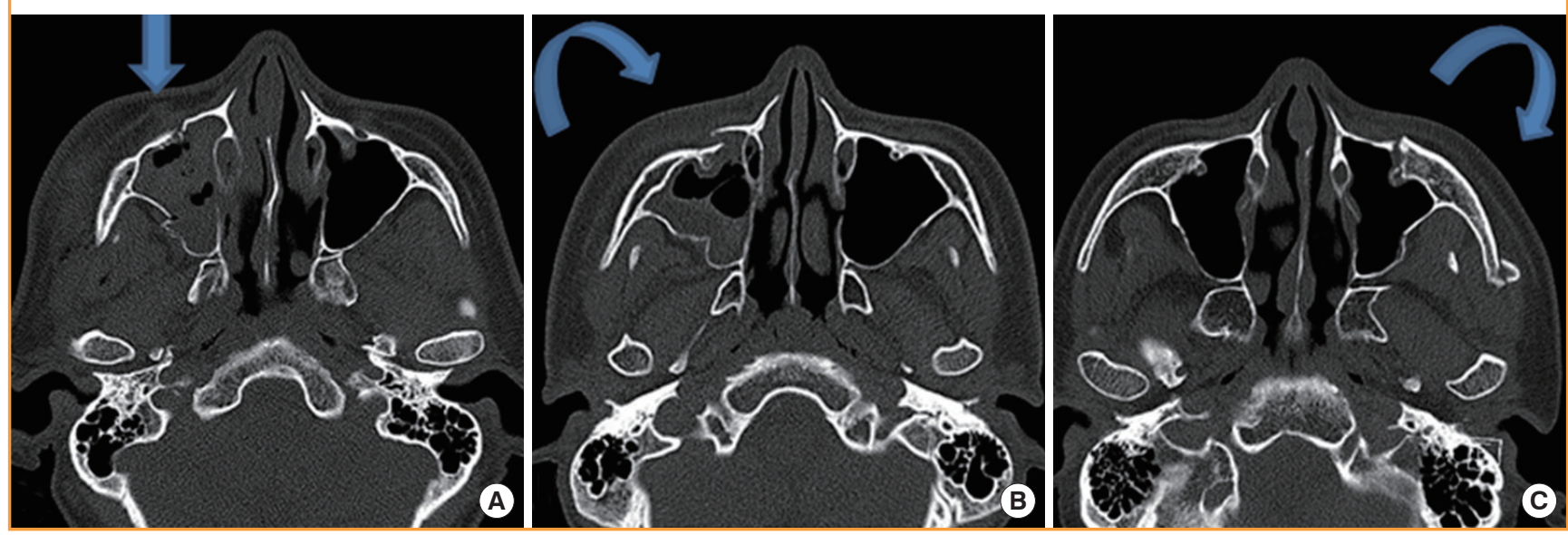
covery period of 0.625 months. The median recovery period of all 33 patients was 0.25 months. There was no statistically significant difference in the trigeminal nerve recovery period between types of fractures (Table 2). Zygomatic fracture patients were classified according to Knight and North classification (Fig. 3). Nine patients were classified into group III, and their median trigeminal nerve recovery period was 1.00 month. Four patients were classified into group IV, and their median recovery period was 1.625 months. Three patients were classified into group V, and their median recovery period was 1.00 month. According to statistical comparison of the recovery period between groups classified by Knight and North classification, there was no statistically significant difference in the trigeminal nerve recovery period among all groups (Table 3 ). Blowout fracture patients were classified according to the site of fracture (Fig. 4). Eight patients had an inferior wall fracture, and their median trigeminal nerve recovery period was 0.25 months. Eight patients had a medial wall fracture, and their median recovery period was 0.625 months. Five patients had a medioinferior wall fracture, and their median recovery period was 0.25 months. Statis-

Table 3 . The trigeminal nerve recovery period according to Knight and North classification of zygomatic fracture by Neurometer CPT test

\begin{tabular}{|lccc|}
\hline $\begin{array}{l}\text { Knight and } \\
\text { North } \\
\text { classification }\end{array}$ & $\begin{array}{c}\text { No. of } \\
\text { patients }\end{array}$ & $\begin{array}{c}\text { Average trigeminal } \\
\text { nerve recovery } \\
\text { period (mo) }\end{array}$ & P-value ${ }^{\text {a) }}$ \\
\hline III & 9 & $1.00(0.25,14.00)$ & 0.926 \\
IV & 4 & $1.625(0.25,6.00)$ & \\
V & 3 & $1.00(0.25,1.00)$ & \\
\hline $\begin{array}{l}\text { Values are presented as median (minimum, maximum). } \\
\text { CPT, current perception threshold. } \\
\text { alkruskal-Wallis test. }\end{array}$ & & \\
\hline
\end{tabular}

tical comparison of the trigeminal nerve recovery period between groups classified according to the site of blowout fracture showed no significant difference (Table 4). The trigeminal nerve recovery period based on Neurometer CPT testing was compared based on each patient's subjective feelings. The median recovery period of blowout fracture patients, according to the Neurometer CPT test, was 0.25 months. According to patients' subjective feelings, the median recovery period was 0.25 months. The median recovery period of zygomatic fracture patients was 1.00 month according to the Neurometer CPT test, and 2.50 months according to patients' subjective feelings. The median recovery period of patients with both fractures was 0.625 months according to the Neurometer CPT test, and 3.00 months according to patients' subjective feelings. Among blowout fracture patients, zygomatic fracture patients, and patients with both fractures, there was no statistically significant difference in the average recovery period according to the Neurometer CPT test and the average recovery period according to patients' subjective feelings (Table 5).

Table 4. The trigeminal nerve recovery period according to the site of blowout fracture by Neurometer CPT test

\begin{tabular}{|lccc|}
\hline $\begin{array}{l}\text { Site of blowout } \\
\text { fracture }\end{array}$ & $\begin{array}{c}\text { No. of } \\
\text { patients }\end{array}$ & $\begin{array}{c}\text { Average trigeminal } \\
\text { nerve recovery } \\
\text { period (mo) }\end{array}$ & P-value \\
\hline Inferior wall & 8 & $0.25(0.25,6.00)$ & 0.898 \\
Medial wall & 8 & $0.625(0.25,3.00)$ & \\
Medioinferior wall & 5 & $0.25(0.25,3.00)$ & \\
\hline $\begin{array}{l}\text { Values are presented as median (minimum, maximum). } \\
\text { CPT, current perception threshold. } \\
\text { alKruskal-Wallis test. }\end{array}$ & \\
\end{tabular}

\section{Fig. 4. The classification of blowout fracture}

The classification of blow-out fracture according to the site of fracture. (A) Medial wall fracture. (B) Inferior wall fracture. (C) Medioinferior wall fracture.
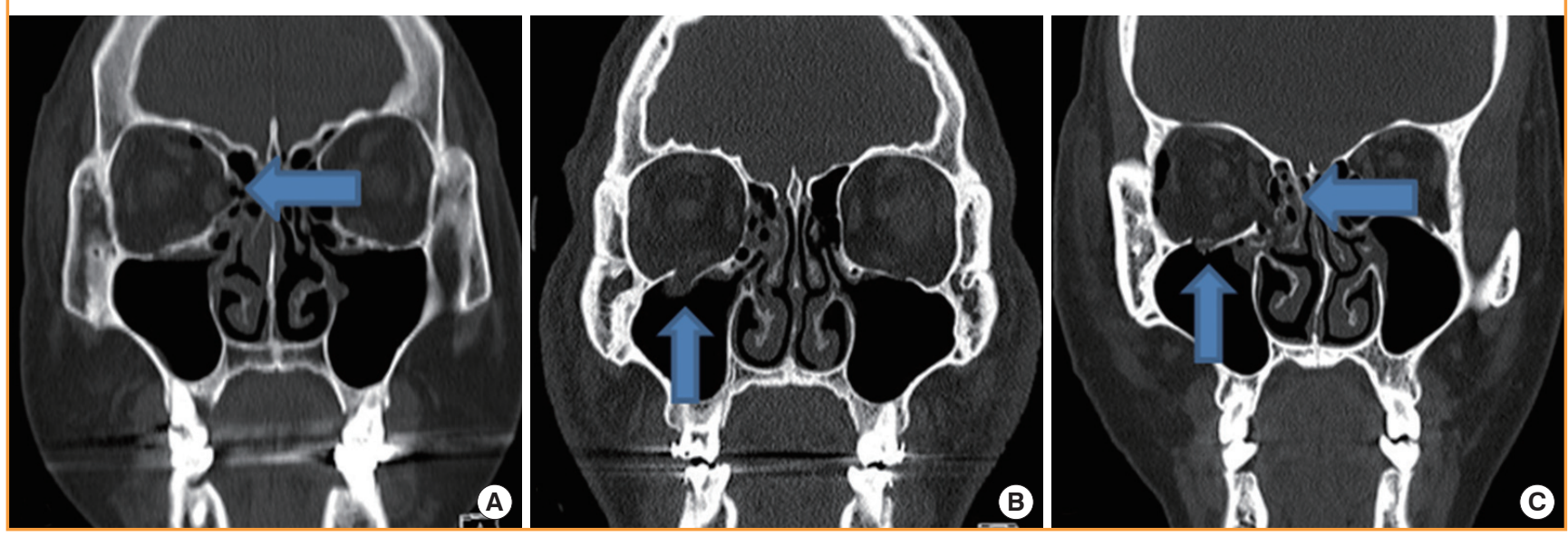
Table 5. Comparison of the trigeminal nerve recovery period according to the Neurometer CPT test and patients' subjective feelings

\begin{tabular}{|c|c|c|c|c|}
\hline Type of fracture & $\begin{array}{l}\text { Trigeminal nerve recovery period } \\
\text { according to the Neurometer } \\
\text { CPT test (mo) }\end{array}$ & $\begin{array}{l}\text { Trigeminal nerve recovery period } \\
\text { according to patients' own } \\
\text { subjective feelings (mo) }\end{array}$ & No. of patients & P-value ${ }^{a)}$ \\
\hline Blowout & $0.25(0.25-6.00)$ & $0.25(0.25,6.00)$ & 17 & $>0.99$ \\
\hline Zygoma & $1.00(0.25-14.00)$ & $2.50(0.25,15.00)$ & 12 & 0.94 \\
\hline Both & $0.625(0.25-3.00)$ & $3.00(2.00,3.00)$ & 4 & 0.25 \\
\hline
\end{tabular}

Values are presented as median (minimum, maximum).

CPT, current perception threshold.

a)Wilcoxon signed-rank test.

\section{DISCUSSION}

The trigeminal nerve is the largest cranial nerve and it divides into 3 branches, that is, the ophthalmic nerve, the maxillary nerve, and the mandibular nerve at the trigeminal ganglion. In case of injury to the trigeminal nerve, hypoesthesia of the lower eyelid, medial cheek, lateral nasal side, and upper lip on the affected side can occur [1].

Facial bone fracture patients often complain of facial hypoesthesia. Surgeons tend to overlook these symptoms, but once they manifest, there is no obvious treatment. These symptoms can lead to a medical conflict for surgeons. First, these symptoms tend to be overlooked, since they are subjective and not visible. Second, there is a lack of objective evidence for a preoperative and postoperative patient's facial sensory status.

Facial sensory nerve injury is evaluated subjectively and objectively. Subjective evaluation is by mechanoreceptor and nociceptor tests. The two-point discrimination test, static light touch, and brush directional stroke are examples of a mechanoreceptor test, and the pinprick test and thermal discrimination test are examples of a nociceptor test. However, these tests have limitations, since they are subjective, and therefore, it is difficult to standardize and quantify them. Objective evaluation is performed by measurement of conduction velocities. However, measurement of conduction velocities requires considerable skill and experience on part of the operator, and expensive equipment, and they are invasive, causing discomfort to the patient [3].

The perception threshold test can diagnose various peripheral sensory neuropathies and estimate therapeutic results according to the minimal intensity of the stimulus that a patient can perceive. There are several types of nerve fibers with different diameters, such as the $\mathrm{A} \beta$ fiber that responds to $2,000-\mathrm{Hz}$ stimulation, the $\mathrm{A} \delta$ fiber that responds to $250-\mathrm{Hz}$ stimulation, and the $\mathrm{C}$ fiber that responds to $5-\mathrm{Hz}$ stimulation. The Neurometer CPT test is one of the perception threshold tests, with a maximum current of $9.99 \mathrm{~mA}$, and it evaluates the peripheral nerve status without causing damage and with minimal influence on the dermal and subcutaneous tissue [4-6]. It can also diagnose early stage peripheral neuropathy, with relatively minimal pain and discomfort, and it can diagnose the status of each peripheral nerve separately. The investigator can perform a simple assessment in a short time period [7-9]. Furthermore, it can be used to evaluate trigeminal neuropathy $[10,11]$ and to diagnose paresthesia such as hyperesthesia or hypoesthesia by assessing fluctuation of the threshold $[12,13]$. The Neurometer CPT test is already being used for nerve status evaluation in various fields [14-16].

Several studies have focused on the evaluation of various peripheral neuropathies using the Neurometer CPT test, but there is a lack of studies that assess the recovery period of peripheral nerves using Neurometer CPT. Okochi et al. [17] studied zygoma fracture patients only and did not evaluate facial sensory status based on the subtype of fracture. Okochi et al. evaluated facial sensory status 1 year and 5 years postoperatively using the Neurometer, but those authors did not assess the facial sensory recovery period [17]. Therefore, the current study is significant in that it deals with the facial sensory recovery period in zygoma and blow-out fracture patients.

The median facial sensory recovery period of all patients in the study was 0.25 months, which was much shorter than expected.

The facial sensory recovery period by fracture type showed no statistically significant differences. Patients with both types of fractures had the second fastest recovery period among the groups. It is difficult to accurately determine why this group had the second fastest recovery period, but the small number of patients with both types of fractures may have influenced the results. Statistical comparison of the trigeminal nerve recovery period between fracture types revealed that there is no statistically significant difference, with a P-value of 0.468 . The facial sensory recovery period in groups classified using the Knight and North classification showed no statistically significant differences. Since groups III, IV, and V differ in the direction of the rotation of the zygoma body, not the degree of injury, the facial sensory recovery period in the groups classified using the Knight and North 
classification may show no statistically significant differences. The facial sensory recovery period in groups classified according to the site of blowout fracture showed no statistically significant differences.

Facial hypoesthesia occurred in 5 patients postoperatively, which suggests that the operation might be the cause of facial hypoesthesia in facial bone fracture patients.

The traditional nerve conduction velocity test can only examine large myelinated nerve fibers. However, a CPT test using the Neurometer is able to evaluate the functional status of three distinct nerve fiber types $(A \beta, A \delta$, and $C)$, so the test is more accurate. In addition, the test is more accurate because an investigator can use false stimuli provided by the Neurometer device. The Neurometer is noninvasive, so it has the advantage of allowing for serial testing [3-6,14].

For paresthesia, the subjective symptoms of patients could be objectively quantified by a Neurometer CPT device. There is a possibility of complaints of facial hypoesthesia postoperatively. Therefore, by evaluating postoperative trigeminal nerve status, operators can compare the patient's postoperative trigeminal nerve status and inform the patients about any damage to the trigeminal nerve. Five patients suffered from facial hypoesthesia postoperatively, which is suggestive of direct intra-operative nerve damage causing facial hypoesthesia in facial-bone fracture patients. However, not all patients had facial hypoesthesia. In our study, 30 patients had normal facial sensory status preoperatively and postoperatively. In addition, when surgeons perform operations that could possibly lead to a medical conflict due to hypoesthesia, a Neurometer CPT test would be useful in preventing and resolving such a conflict.

Hypoesthesia in facial bone fracture patients could be associated with secondary gain. In such cases, a diagnosis is more significant when tests are conducted serially. Nerve conduction velocity tests are too invasive to conduct serially, as they make patients uncomfortable. The Neurometer can be used to conduct serial tests, and also to evaluate small unmyelinated fibers [3-6]. In addition, if a patient with facial hypoesthesia undergoes a test preoperatively, it is helpful for the patient to obtain a disability judgment, and surgeons can protect themselves. In the author's hospital, there were some cases in which patients continued to complain of facial hypoesthesia even though the author adequately explained the possibility that the symptom would persist postoperatively. When the author has access to a physical judgment of blow-out and zygoma fracture patients complaining of facial hypoesthesia, it is difficult to compare postoperative with preoperative symptoms when no preoperative testing is performed. Therefore, we conducted serial Neurometer tests preoperatively and postoperatively, and this study was carried out.
In zygoma fracture patients, the subjective recovery period is longer than that measured by the Neurometer test. However, there was no statistical difference in the average recovery period as measured by the test and the subjective recovery period, so we can conclude that the test is reliable. The Neurometer test is more meaningful than a patient's subjective reports, since the Neurometer test can quantify the degree of injury and provide an objective basis for facial sensory loss.

The limitation of this study is that only a small number of patients were included. Errors could have occurred due to the different sensitivities of individual patients, the amount of gel applied, and the degree of electrode attachment to the face. Therefore, to reduce such errors, using a mean result from repetitive examination is preferable. The test depends on a patient's subjective state. Therefore, to make the results more objective, an investigator can use false stimulation provided by the Neurometer while the patient is unaware of the procedure being performed, and can conduct serial tests until facial sensation is recovered. To evaluate peripheral sensory nerves more objectively, various physical and perceptive examinations, and multifactorial analysis are required. A sufficient number of cases and long-term follow-up are also required.

The Neurometer CPT test is easy to use, non-invasive, and useful in improving the quality of patient management. Facial hypoesthesia, which is a subjective patient symptom, was objectively evaluated with a Neurometer CPT test.

\section{REFERENCES}

1. Neligan P. Plastic surgery. 3rd ed. New York: Elsevier Saunders; 2013.

2. Neurotron Incorporated. Neurometer clinical and research update [Internet]. Baltimore: Neurotron Incorporated; c2016 [cited 2016 Jun 27]. Available from http://www.neurotron. com/BOOKLET_2010-2011.pdf.

3. Poort LJ, van Neck JW, van der Wal KG. Sensory testing of inferior alveolar nerve injuries: a review of methods used in prospective studies. J Oral Maxillofac Surg 2009;67:292300.

4. Dotson RM. Clinical neurophysiology laboratory tests to assess the nociceptive system in humans. J Clin Neurophysiol 1997; 14:32-45.

5. Katims JJ. Electrodiagnostic functional sensory evaluation of the patient with pain: a review of the neuroselective current perception threshold and pain tolerance threshold. Pain Dig 1998;8:219-30.

6. Takekuma K, Ando F, Niino N, et al. Age and gender differences in skin sensory threshold assessed by current percep- 
tion in community-dwelling Japanese. J Epidemiol 2000; 10:S33-8.

7. Weseley SA, Sadler B, Katims JJ. Current perception: preferred test for evaluation of peripheral nerve integrity. ASAIO Trans 1988;34:188-93.

8. Katims JJ, Naviasky EH, Ng LK, et al. New screening device for assessment of peripheral neuropathy. J Occup Med 1986; 28:1219-21.

9. Chado HN. The current perception threshold evaluation of sensory nerve function in pain management. Pain Dig 1995; 5:127-34.

10. Kim HS, Kho HS, Kim YK, et al. Reliability and characteristics of current perception thresholds in the territory of the infraorbital and inferior alveolar nerves. J Orofac Pain 2000; 14:286-92.

11. Chung JW. The effects of transcutaneous electrical nerve stimulation and electroacupuncture stimualtion therapy on the current perception threshold of orofacial region. J Oral Med Pain 1999;24:301-13.

12. Masson EA, Boulton AJ. The Neurometer: validation and comparison with conventional tests for diabetic neuropathy.
Diabet Med 1991;8 Spec No:S63-6.

13. Masson EA, Veves A, Fernando D, et al. Current perception thresholds: a new, quick, and reproducible method for the assessment of peripheral neuropathy in diabetes mellitus. Diabetologia 1989;32:724-8.

14. Matsutomo R, Takebayashi K, Aso Y. Assessment of peripheral neuropathy using measurement of the current perception threshold with the neurometer in patients with type 2 diabetes mellitus. J Int Med Res 2005;33:442-53.

15. Oishi M, Mochizuki Y, Suzuki Y, et al. Current perception threshold and sympathetic skin response in diabetic and alcoholic polyneuropathies. Intern Med 2002;41:819-22.

16. Jeon JW, Kim KH, Ko MY, et al. Effect of Nd:YAG laser irradiation to mental region for sensory nerve conduction change. J Oral Med Pain 2005;30:447-55.

17. Okochi M, Ueda K, Mochizuki Y, et al. How can paresthesia after zygomaticomaxillary complex fracture be determined after long-term follow-up? A new and quantitative evaluation method using current perception threshold testing. J Oral Maxillofac Surg 2015;73:1554-61. 